\title{
Polarographic and spectroscopic studies of the effects of fluoroacetate/fluorocitrate on cells and mitochondria
}

\author{
Valeriy P. Zinchenko ${ }^{\text {a }}$, Nikolay V. Goncharov ${ }^{\mathrm{b}}$, Vera V. Teplova ${ }^{\mathrm{c}}$, Vitaliy A. Kasymov ${ }^{\mathrm{a}}$, \\ Olga I. Petrova ${ }^{a}$, Alexey V. Berezhnov ${ }^{a}$, Evgeniy V. Senchenkov ${ }^{b}$, Igor V. Mindukshev ${ }^{d}$, \\ Richard O. Jenkins ${ }^{\mathrm{e}, *}$ and Andrey S. Radilov ${ }^{\mathrm{b}}$ \\ ${ }^{a}$ Institute of Cell Biophysics, Russian Academy of Sciences, Pushchino, Russia \\ ${ }^{\mathrm{b}}$ Research Institute of Hygiene, Occupational Pathology and Human Ecology, St-Petersburg, Russia \\ ${ }^{\mathrm{c}}$ Institute of Theoretical and Experimental Biophysics, Russian Academy of Sciences, Pushchino, \\ Russia \\ ${ }^{\mathrm{d}}$ I.M. Sechenov Institute of Evolutionary Physiology and Biochemistry, Russian Academy of Sciences, \\ St-Petersburg, Russia \\ ${ }^{\mathrm{e}}$ School of Allied Health Sciences, De Montfort University, Leicester, UK
}

\begin{abstract}
Experiments were performed with rat liver mitochondria, Ehrlich ascite tumor cells (EATC) and cardiomyocytes, exposed to fluoroacetate (FA) or fluorocitrate (FC) in vitro. The effects of FA developed at much higher concentrations in comparison with FC and was dependent upon respiratory substrates: with pyruvate, FA induced a slow oxidation of pyridine nucleotides $(\mathrm{NAD}(\mathrm{P}) \mathrm{H})$ and inhibition of respiration. $\mathrm{NAD}(\mathrm{P}) \mathrm{H}$ oxidation was prevented by incubation of mitochondria with cyclosporin A (CsA), an inhibitor of mitochondrial permeability transition pore. Studies of the NAD(P)H level and calcium response generated in EATC under activation with ATP via the metabotropic P2Y receptor, revealed a loss of NAD(P)H from mitochondria resulting in a shift in the balance of mitochondrial and cytosolic NAD $(\mathrm{P}) \mathrm{H}$ on exposure to FA. An increase of cytosolic $\left[\mathrm{Ca}^{2+}\right]$ was observed in the cell lines exposed to FA and is explained by activation of plasma membrane calcium channels; this mechanism could have an impact on amplitude and rate of $\mathrm{Ca}^{2+}$ waves in cardiomyocytes, and cause the hypersensitivity of platelets reported on earlier. Highlighting the reciprocal relationship between intracellular NAD(P)H and calcium balance, we discuss metabolic pathway modulation in the context of development of an effective therapy for FA poisoning.

Keywords: Fluoroacetate, intracellular calcium, pyridine nucleotides, mitochondria, membrane potential
\end{abstract}

\section{Introduction}

Fluoroacetates (FA) are known to be chemical compounds of the general formula $\mathrm{CH}_{2} \mathrm{FCOOR}$. The toxic action of FA is fairly well understood, though no appreciable progress in developing an effective therapy has been achieved for the past six decades [1]. The conversion of FA into fluorocitrate (FC) leads to inhibition of mitochondrial aconitase followed by accumulation of citrate [2]. Calcium chelation is one of the main effect of citrate accumulation; concomitant decrease in the concentration of parathyroid hormones probably further decreases the concentration of plasma calcium under intoxication with

\footnotetext{
${ }^{*}$ Corresponding author: R.O. Jenkins, School of Allied Health Sciences, De Montfort University, Leicester, LE1 9BH, UK. Tel.: +44 116 2506306; E-mail: roj@dmu.ac.uk.
} 
FA [3]. Enhanced urinary excretion of $\mathrm{Ca}^{2+}$ has also been observed during citrate accumulation [3]. The degree of hypocalcemia correlates with prolongation of the Q-T interval in electrocardiogram, which is a consequence of a broad range of cardiac arrhythmias. Decrease in calcium level may be responsible for the hypocalcemic tetanus [4] that manifests itself in typical convulsions, blood clotting lesions and hypotension leading to vascular collapse and death [5]. Our recent experiments with FA in vitro and in vivo have shown that rat blood platelets become primarily hypersensitive to ADP with aggregation developing at very low ADP concentrations of $10-15 \mathrm{nM}$, followed by their transition to a refractory (insensitive) state [6].

Despite a great deal of investigative study on the intracellular events under FA intoxication, there is only vague understanding of intracellular calcium disturbances. The increase in cellular citrate level affects glucose metabolism through inhibition of phosphofructokinase [7], while in rat hepatocytes accumulating citrate also inhibits guanylate cyclase [8]. In adipose tissue, FA causes reduction in the basal and hormone-stimulated cAMP levels by inhibition of adenylate cyclase and has no effect on the activity of cAMP phosphodiesterase [9]. A negative modulation of gluconeogenesis in hepatocytes of poisoned animals has been reported [10]. Both in vivo and in vitro experiments have shown that acute FA poisoning decreases the ATP level in heart and other organs and tissues [7,11]. The changes in ATP concentration were not associated with uncoupling of respiration and phosphorylation [12]. Under intoxication with FA, structural changes of various organelles have been observed, especially in mitochondria [13]. FA caused abnormalities in the matrix of mitochondria that developed over several minutes, being apparent by their swelling and loss of electronic density; this damage was explained by an increase in osmotic pressure resulting from citrate accumulation [14]. In our early experiments with rat liver mitochondria in vitro [15], FC completely inhibited endogenous oxygen uptake (metabolic state $\mathrm{v}_{1}$ ). ATP synthesis was also inhibited and the redox state of pyridine nucleotides was assumed to be the primary cause of the mitochondrial respiration disturbances. However, there is some evidence that the relevant parameters and functions are not affected under action of FA in cells in vitro. For example, FA did not affect ATP, GTP and cyclic nucleotides in hepatocytes [8]. Furthermore, mitochondria isolated from various tissues of rats intoxicated with FA have been shown to disproportionately change their respiratory activity $[2,14]$. Probably of importance to such effects are the relative activities of various metabolic pathways in different tissues and cells: glycolysis $v s$ pentose phosphate pathway; pyruvate dehydrogenase $v s$ thiokinase route of carbon entrance to the TCA cycle; transamination vs glutamate dehydrogenase anaplerotic pathways; mitochondrial $v s$ cytosolic aconitase for citrate oxidation, etc.

The interrelation of signalling and metabolic pathways during FA intoxication is poorly understood. Inhibition of aconitase may lead to both inhibition of the TCA cycle and citrate accumulation, and the intensity or balance of these processes is different in various cell types. Consequently, it is very difficult to establish the primary reaction of different cell types, and more so for the whole organism. Improved understanding would promote development of an effective therapy for FA and other metabolic poisons, as well as for hypoxic and reoxigenation states. The aim of the research reported on here was to further reveal mechanisms involved in disturbances of calcium balance and pyridine nucleotides under exposure to FA or FC.

\section{Materials and methods}

All experiments were carried out in accordance with USA guidelines on care and use of laboratory animals [16]. 


\subsection{Reagents}

Sodium fluoroacetate (FA) was synthesized in-house at RIHOPHE. Other reagents were obtained from: Sigma - Dulbeco's Modified Eagle's Medium (DMEM), Hanks' buffer saline solution (HBSS), Fetal Bovine Serum (FBS), ADP, ATP, FC; Merck - sodium acetate; ICN - HEPES, rotenone, oligomicin; Fluka - cyclosporin A (CsA), rotenone, oligomicin, carbonyl cyanide 4-trifluoromethoxyphenylhydrazone (FCCP), chlortetracycline (CTC); Calbiochem - digitonin; Molecular Probes - Fluo-4 acetoxymethyl ester (Fluo-4AM), Indo-1AM, tetramethylrhodamine methyl ester (TMRM), Pluronic; Reakhim, Russia - salts and glucose.

\subsection{Isolation and study of rat liver mitochondria}

The experiments were performed with mitochondria isolated from rat liver [17]. Respiratory characteristics of the isolated mitochondria were obtained by polarography [18]. The measurements were performed in a temperature-controlled cell $\left(37^{\circ} \mathrm{C}\right)$ with a built-in oxygen electrode. Mitochondria were incubated in a medium containing $\left(\mathrm{mM}\right.$ ): $\mathrm{KCl}-120, \mathrm{KH}_{2} \mathrm{PO}_{4}-2$, HEPES - 10 (pH adjusted to 7.4 with Trizma base), and respiratory substrates. To study the state of pyridine nucleotides, NAD(P)H fluorescence was measured using a Hitachi F4000 fluorometer, with excitation wavelength $340 \mathrm{~nm}$ and emission wavelength $460 \mathrm{~nm}$.

\subsection{Isolation of rat cardiomyocytes}

Cardiomyocytes were isolated from Wistar rats of 150-180 g using collagenase solution containing the following components $(\mathrm{mM}): \mathrm{NaCl}-120, \mathrm{KCl}-5.8, \mathrm{MgSO}_{4}-1.5, \mathrm{KH}_{2} \mathrm{PO}_{4}-1.4, \mathrm{NaHCO}_{3}-4.3$, glucose - 14, and HEPES - $10\left(\mathrm{pH} \mathrm{7.0)}\right.$ at $37^{\circ} \mathrm{C}$, as described by Powell et al. [19]. The viability of the calcium-tolerant rectangular cells obtained was $85-90 \%$. The suspension of cardiomyocytes was placed in a Petri dish coated with collagen. After $60 \mathrm{~min}$, unattached cells were washed out. The attached cells were used in experiments.

\subsection{Measurements of cytosolic calcium $\left[\mathrm{Ca}^{2+}\right]_{i}$ and mitochondrial membrane potential $\Delta \Psi-m$ using confocal laser scanning microscopy}

Before measurements of $\left[\mathrm{Ca}^{2+}\right]_{\mathrm{i}}$ cardiomyocytes were incubated for $40 \mathrm{~min}$ at $37^{\circ} \mathrm{C}$ with $5 \mu \mathrm{M}$ Fluo$4 \mathrm{AM}$ and $0.005 \%$ Pluronic in HBSS. For simultaneous measurements of $\left[\mathrm{Ca}^{2+}\right]_{\mathrm{i}}$ and $\Delta \Psi-\mathrm{m}, 20 \mathrm{nM}$ TMRM (fluorescent probe for $\Delta \Psi-\mathrm{m}$ ) was added $15 \mathrm{~min}$ before the end of incubation. At such low TMRM concentration accumulation of fluorescent probe is accompanied by fluorescence enhancement with increasing potential. The cells were washed with three aliquots of HBSS, and the Petri dish was mounted on the sample stage of Carl Zeiss LSM 510 confocal laser scanning microscope. The working volume was $1.5 \mathrm{ml}$. The measurements were performed with the Zeiss PlanNeofluar $\times 20$ air-immersion lens and the Zeiss PlanNeofluar $\times 63$ water-immersion lens. Fluo4 fluorescence was excited with an Ar laser $(488 \mathrm{~nm})$ and registered with a BP500-550 nm cut-off filter. To measure the mitochondrial membrane potential, TMRM fluorescence was excited with a He/Ne laser $(543 \mathrm{~nm})$ and registered with an LP560 nm transmitting filter. Image processing and analysis was performed using Kinetic Imaging software (Liverpool, UK) and OriginPro 7.0 programs. 


\subsection{Isolation of Ehrlich ascite tumor cells (EATC); measurements of pyridine nucleotides, cytosolic $\left[\mathrm{Ca}^{2+}\right]_{i}$ and endoplasmic reticulum $[\mathrm{Ca}]_{\text {er }}$ in cells suspensions}

EATC were isolated on the 7th or 8th day after peritoneal transplantation into the NMRI/BÀLB mice [20]. The cells $\left(2-5 \times 10^{7} / \mathrm{ml}\right)$ were incubated at $37^{\circ} \mathrm{C}$ for $40 \mathrm{~min}$ in Hanks' medium containing $10 \mathrm{mM}$ HEPES at pH 7.2 with $2 \mu \mathrm{M}$ indo-1AM and $0.005 \%$ Pluronic F127. The cells were then washed twice with a fresh medium, resuspended to the same cell concentration in the dye-free medium and were used within an hour in the experiments. To monitor $\left[\mathrm{Ca}^{2+}\right]_{\mathrm{i}}$ changes, the cells were placed at a concentration of $5 \times 10^{6} / \mathrm{ml}$ into the spectrofluorometer chamber equipped with a magnetic stirrer and thermostatically controlled at $28^{\circ} \mathrm{C}$ in Hanks' medium containing $(\mathrm{mM}): \mathrm{NaCl}-140, \mathrm{KCl}-5.4, \mathrm{CaCl}_{2}-1.3, \mathrm{MgSO}_{4}-1$, $\mathrm{KH}_{2} \mathrm{PO}_{4}-1, \mathrm{Na}_{2} \mathrm{HPO}_{4}, \mathrm{NaHCO}_{3}-1$, glucose - 6, HEPES - 20 (pH 7.4). The excitation wavelength was $365 \mathrm{~nm}$, the emission were measured in $405 / 490 \mathrm{~nm}$. $\mathrm{Ca}^{2+}$-sensitive chlortetracycline (CTC) fluorescence was used to register $\mathrm{Ca}^{2+}$ changes in the endoplasmic reticulum. In these experiments, the cell suspensions were incubated for $40 \mathrm{~min}$ in the presence of $12 \mu \mathrm{M}$ of CTC at $37^{\circ} \mathrm{C}$. Then, the CTC-loaded cells were placed into the spectrofluorometer chamber without preliminary washing. The excitation and emission wavelengths were $405 \mathrm{~nm}$ and $530 \mathrm{~nm}$, respectively. The endogenous $\mathrm{NAD}(\mathrm{P}) \mathrm{H}$ fluorescence was measured at $365 \mathrm{~nm}$ excitation and $465 \mathrm{~nm}$ emission wavelengths.

\section{Results}

\subsection{Effects of FA on the functional characteristics of isolated rat liver mitochondria}

The effects of FA developed at much higher concentrations in comparison with those of FC and depended on the type of respiratory substrates. Figure 1 shows the effects of FA on redox state of pyridine nucleotides and oxidative phosphorylation of rat liver mitochondria with pyruvate as respiratory substrate. FA at a concentration of $4 \mathrm{mM}$ induced slow oxidation of pyridine nucleotides and inhibition of oxidative phosphorylation (Fig. 1A). The time span of oxidative phosphorylation and the rate of pyridine nucleotides oxidation increased linearly with increasing FA concentrations in the medium (Fig. 1B). An inhibition of ADP-induced and, especially, uncoupled respiration of rat liver mitochondria was observed. Figure 2 shows dependences of uncoupled respiration of mitochondria upon concentrations of FC and FA: the $\mathrm{EC}_{50}$ concentrations differed by a thousandfold. These data are consistent with inhibition of oxidative phosphorylation demonstrated under exposure to FC, and arises through inhibition of the respiratory chain [15]. In the presence of succinate as respiratory substrate a very small (if any) change in mitochondrial functions was observed under exposure to FA (data not shown here). Also, FA at concentrations up to $16 \mathrm{mM}$ did not affect oxidative phosphorylation in mitochondria respiring on glutamate plus malate.

Figure 3 demonstrates that the effect of FA $(10 \mathrm{mM})$ on pyridine nucleotides oxidation was prevented by incubation of mitochondria with CsA, an inhibitor of mitochondrial permeability transition pore. This indicates that under the action of FA the pyridine nucleotides could leak from mitochondria through the permeability transition pore; the process of pore opening is reversible and prevention of oxidation and/or leakage of pyridine nucleotides from mitochondria can help to return them to the normally functioning state. 

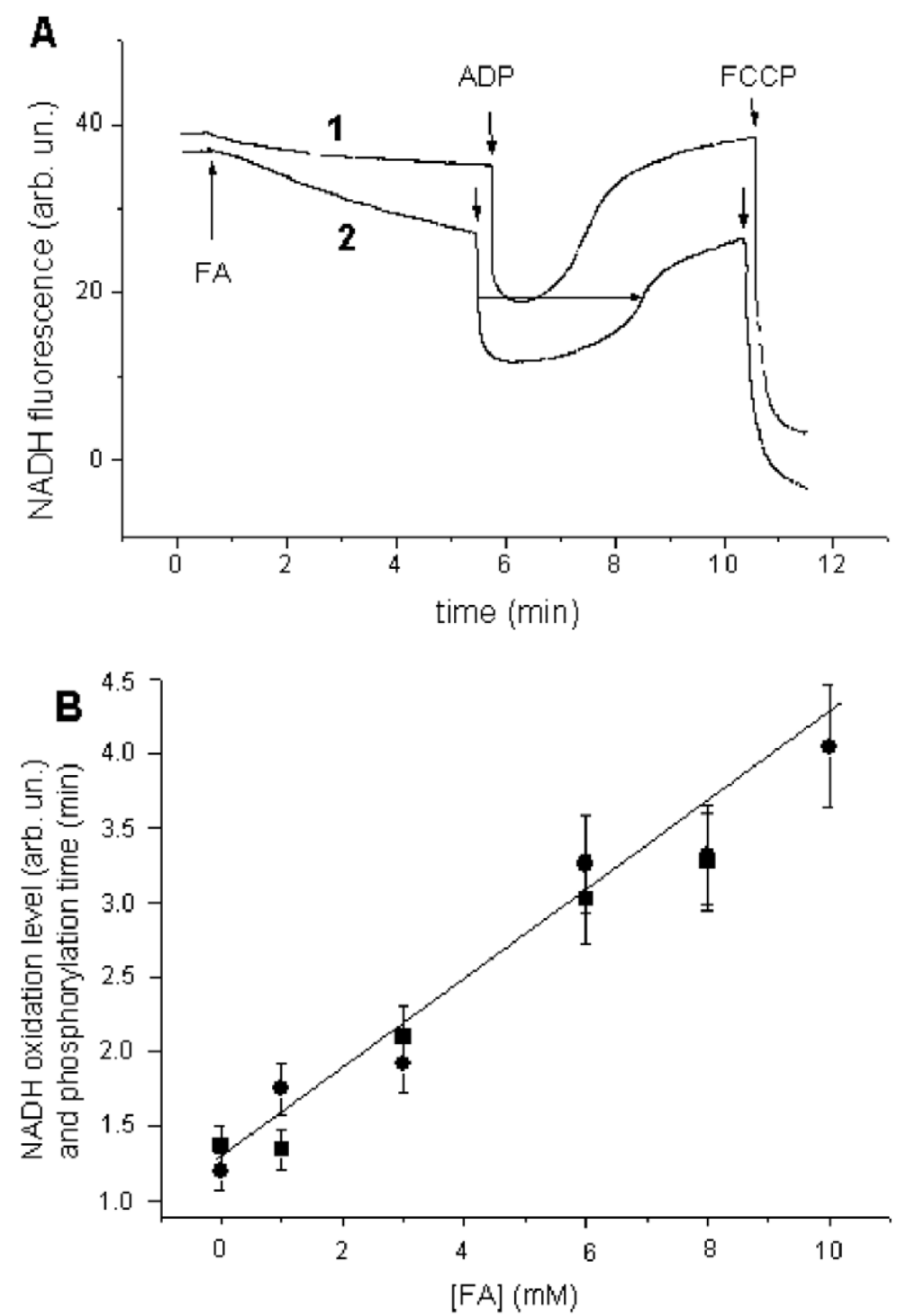

Fig. 1. Effects of FA on respiration of rat liver mitochondria. (A) FA influence (trace 2) on oxidative phosphorylation and redox state of pyridine nucleotides with pyruvate as respiratory substrate. Trace 1 - control. Incubation medium contained (mM): $\mathrm{KCl}-120 ; \mathrm{KH}_{2} \mathrm{PO}_{4}-2$; HEPES - $10(\mathrm{pH} 7.2)$; pyruvate - 5; mitochondrial protein - $1 \mathrm{mg} / \mathrm{ml}$. FA - $4 \mathrm{mM}$; ADP - $120 \mu \mathrm{M}$; FCCP $-\mu$ M. (B) Dependence of the time of phosphorylation (-) and level of NADH oxidation ( $\mathbf{\square})$ upon concentration of FA. Substrates: pyruvate plus malate; both measured parameters are proportional to FA concentration and reflect inhibition of respiration at the level of TCA cycle. Summarized data: means $\pm 10 \%$ error $(n=5)$.

\subsection{Effects of FA on Ehrlich ascite tumor cells (EATC)}

On completion of experiments with rat liver mitochondria, we supposed that the effects of FA on isolated cells would primarily involve the mitochondrial membrane potential, level of $\mathrm{NAD}(\mathrm{P}) \mathrm{H}$, and intracellular $\left[\mathrm{Ca}^{2+}\right]$ level. Membrane potential measurements were carried out with the TMRM fluorescent probe at low concentrations, when probe accumulation in mitochondria and their increasing membrane 


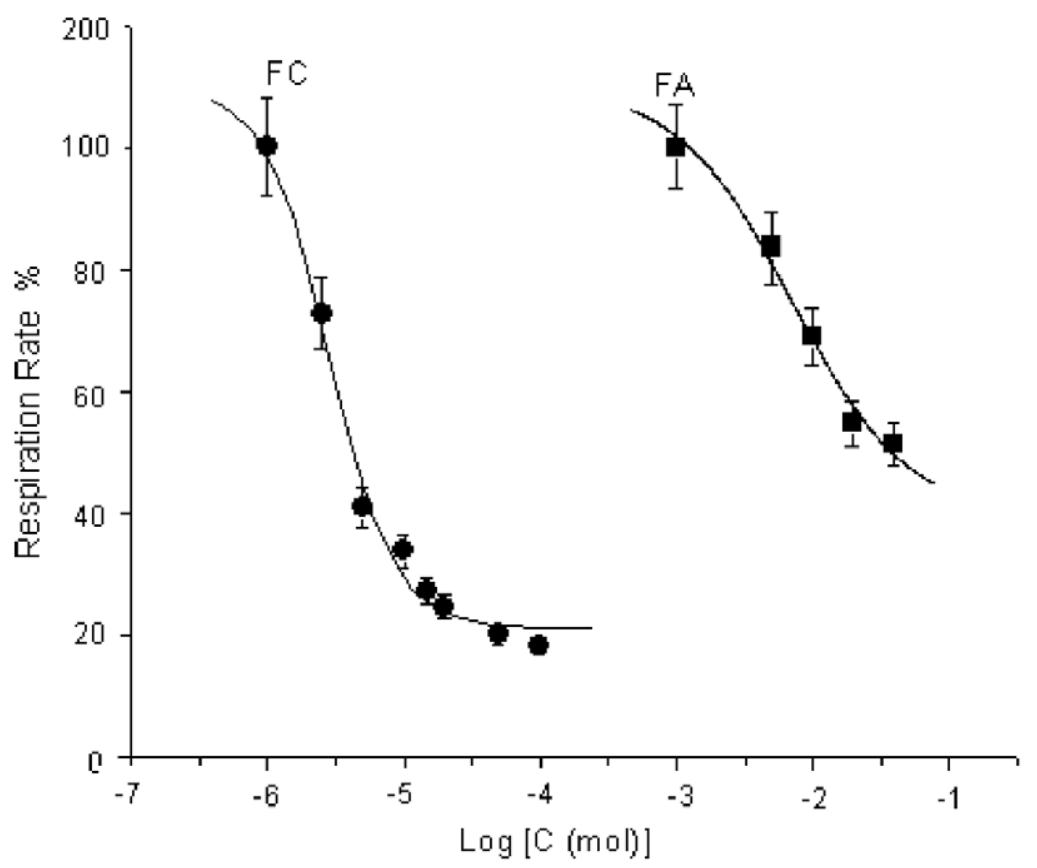

Fig. 2. Dependence of the respiration rate of mitochondria upon exposure to FC or FA in the presence of FCCP. Substrates: pyruvate plus malate. $\mathrm{EC}_{50} 7-10 \mu \mathrm{M}$ and $7-13 \mathrm{mM}$ for FC and FA, respectively. Summarized data: means $\pm 8 \%$ error $(n=6)$.

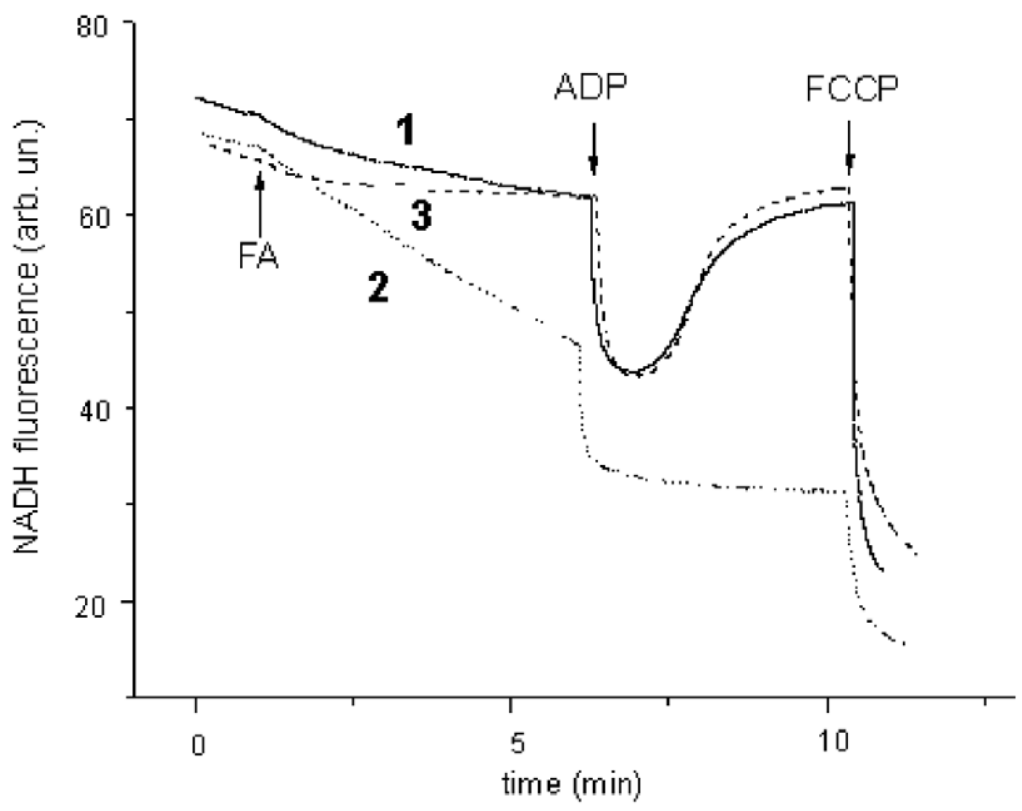

Fig. 3. Effect of FA on redox state of pyridine nucleotides and oxidative phosphorylation of rat liver mitochondria (pyruvate as respiratory substrate) and prevention of this effect by CsA. Incubation medium was as described for Fig. 1. Traces: (1) control experiment (black line); (2) $10 \mathrm{mM} \mathrm{FA}$ (dotted line); (3) $10 \mathrm{mM} \mathrm{FA}$ in the presence of $1 \mu \mathrm{M} \mathrm{CsA}$ (dashed line). ADP - 120 $\mu \mathrm{M}$; $\mathrm{FCCP}-1 \mu \mathrm{M}(n=4)$. 


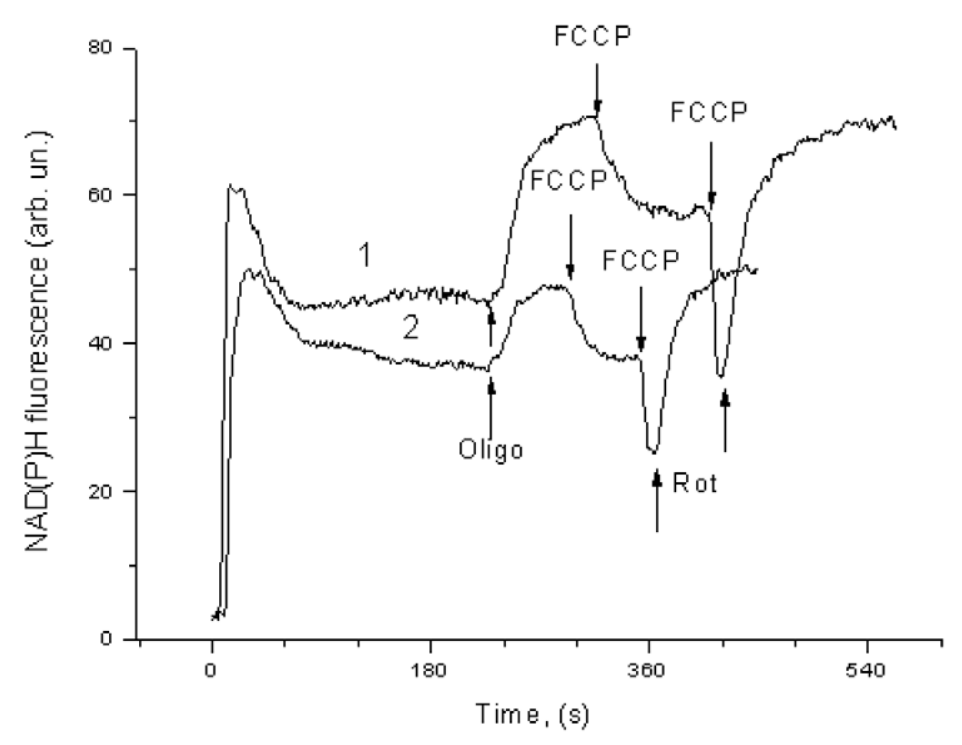

Fig. 4. Changes in fluorescence of NAD(P)H in EATC in response to oligomycin $(1 \mu \mathrm{g} / \mathrm{ml})$, FCCP $(5 \mathrm{nM}$ and $1 \mu \mathrm{M})$, and rotenone $(1 \mu \mathrm{g} / \mathrm{ml})$. Traces: (1) control; (2) exposure to FA, $30 \mathrm{mM}\left(3.5\right.$ hours of incubation at $\left.28^{\circ} \mathrm{C}\right)(n=4)$.

potential was accompanied by fluorescence enhancement.

As evident from the above, exposure to FA results in inhibition of the TCA cycle, slow oxidation of $\mathrm{NAD}(\mathrm{P}) \mathrm{H}$, and inhibition of respiration and oxidative phosphorylation. We expected the redox state of mitochondrial pyridine nucleotides to be the most sensitive parameter under exposure to FA. Zinchenko et al. [21] demonstrated that about $80 \%$ of the cells' natural fluorescence under excitation of $350 \mathrm{~nm}$ and emission of $470 \mathrm{~nm}$ was caused by its mitochondrial $\mathrm{NAD}(\mathrm{P}) \mathrm{H}$. We studied the pyridine nucleotides level and calcium response generated in EATC under activation with ATP via the metabotropic P2Y receptor. Since FA addition did not evoke immediate changes of NAD(P)H fluorescence in the EATC (data not shown here), we investigated the cells after a long-term incubation with FA (2-5 hours). Figure 4 shows changes in fluorescence of $\mathrm{NAD}(\mathrm{P}) \mathrm{H}$ in control cells and in cells incubated with $30 \mathrm{mM}$ of FA for 3.5 hours in response to inhibition of pyridine nucleotides by oligomycin, partial uncoupling of mitochondria with various doses of FCCP, and inhibition of NADH oxidation with rotenone. The level of fluorescence was lower in the presence of FA as compared with control cells. The initial drop in fluorescence was caused by transference of the cells from cold into oxygen-enriched medium at $28^{\circ} \mathrm{C}$ and reflects an enhancement of ATPase activity. Thereafter, the level of fluorescence was stable in control cells. Under exposure to FA the fluorescence slowly decreased, which is in agreement with the experiments on isolated mitochondria and is evidently caused by a partial inhibition of the TCA cycle. Inhibition of oxidative phosphorylation with oligomycin only slightly reduced $\mathrm{NAD}(\mathrm{P}) \mathrm{H}$ in the cells intoxicated with FA, indicating a pre-existing inhibition of oxidative phosphorylation and decrease of the membrane potential of mitochondria caused by FA. Introduction of low quantities of uncoupling agent FCCP $(5 \mathrm{nM})$ partially oxidises $\mathrm{NAD}(\mathrm{P}) \mathrm{H}$, the relative level of oxidation in the presence of FA being greater, which also indicates a pre-existing inhibition of the TCA cycle of mitochondria that apparently should be the cause of the above mentioned inhibition of oxidative phosphorylation. The complete oxidation of the mitochondrial $\mathrm{NAD}(\mathrm{P}) \mathrm{H}$ with FCCP followed by its reduction with rotenone revealed a total decrease of this mitochondrial $\mathrm{NAD}(\mathrm{P}) \mathrm{H}$ pool in the cells incubated with FA. This could indicate a loss 


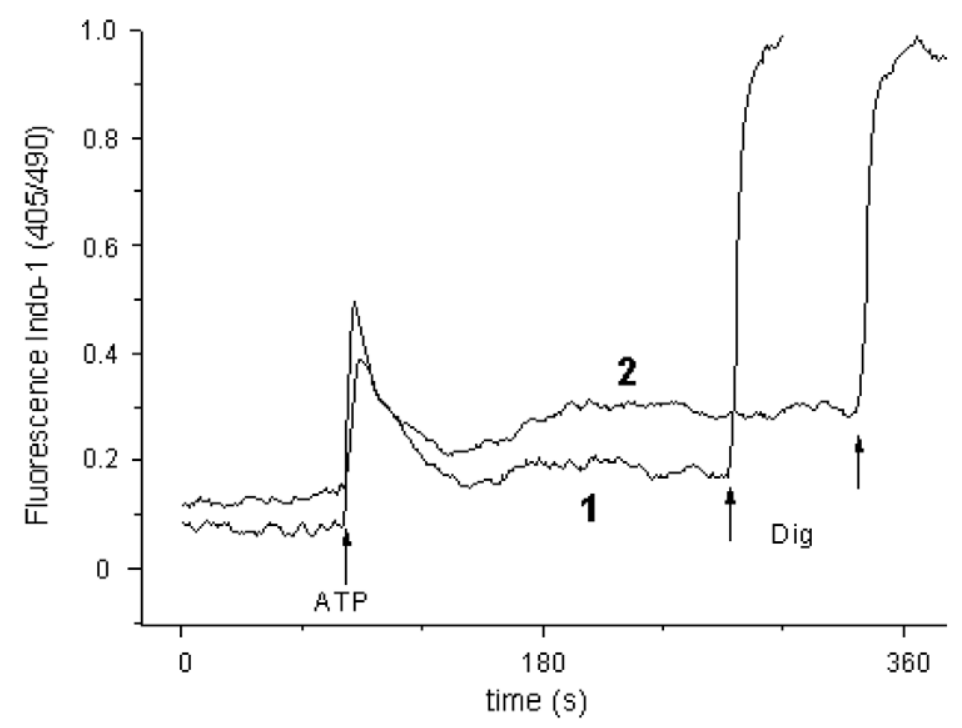

Fig. 5. Changes of cytosolic $\left[\mathrm{Ca}^{2+}\right]$ in EATC under activation of the purinoceptors with ATP, $10 \mu \mathrm{M}$. Traces: (1) control cells; (2) cells incubated for 2.5 hours with FA, $10 \mathrm{mM}(n=4)$.

or leakage of pyridine nucleotides from mitochondria resulting in a shift in the balance of mitochondrial and cytosolic $\mathrm{NAD}(\mathrm{P}) \mathrm{H}$.

The initial level of cytosolic $\left[\mathrm{Ca}^{2+}\right]_{\mathrm{i}}$ was slightly elevated in the cells incubated for 2.5 hours with FA at $10 \mathrm{mM}$ (Fig. 5). The level of the plateau after ATP-induced $\mathrm{Ca}^{2+}$ impulse was also increased; an effect observed with FA at concentrations 5, 10, 15 and $20 \mathrm{mM}$. Exposure to FA probably resulted in depletion of intracellular calcium stores and activation of extracellular $\mathrm{Ca}^{2+}$ entry via store-operated calcium channels (SOC channels). Using CTC fluorescence for registration of $\mathrm{Ca}^{2+}$ in ER, we revealed that ATP produced cyclic changes of $\mathrm{Ca}^{2+}$ concentration in these organelles. The ER was firstly emptied of $\mathrm{Ca}^{2+}$ and then slowly refilled (Fig. 6). Figure 6 shows changes in concentration of $\mathrm{Ca}^{2+}$ in endoplasmic reticulum of EATC after ATP addition $(4.5 \mu \mathrm{M})$ in control experiment (trace 1) and under exposure to 10 and $20 \mu \mathrm{M}$ FC (traces 2 and 3, respectively). FC slightly increased the amplitude of the $\mathrm{Ca}^{2+}$ efflux and rate of its return into ER. In another experiment, the cells were exposed to $20 \mathrm{mM}$ FA or SA (sodium acetate) for 2.5 hours. The response of EATC to ATP $(4.5 \mu \mathrm{M})$ was then registered. As in the case of FC, FA enhances the amplitude of the response and rate of return for $\mathrm{Ca}^{2+}$ into ER (data not shown here).

It should be noted that the period of endoplasmic reticulum refilling with $\mathrm{Ca}^{2+}$ is rather long (8$10 \mathrm{~min}$ ) in normal conditions, indicating that after mobilization of intracellular $\mathrm{Ca}^{2+}$ and its exit from endoplasmic reticulum to cytosol the plasma membrane Ca-ATPase quickly pumps the calcium ions out from cells, thus markedly reducing the cytosolic $\mathrm{Ca}^{2+}$ concentration at which calcium ions are being transported back into endoplasmic reticulum. As has been previously demonstrated [22], the rate of endoplasmic reticulum refilling with $\mathrm{Ca}^{2+}$ after its mobilization is limited by entry of extracellular $\mathrm{Ca}^{2+}$ into cells, i.e. by activity of calcium channels of PM. Thus, by measuring the rate of ER refilling with $\mathrm{Ca}^{2+}$ it is possible to speculate about the activity of the plasma membrane calcium channels. Accordingly, the data obtained in our experiments indicate that FA can activate the entry of $\mathrm{Ca}^{2+}$ into cells via SOC channels. Thus, an increase of cytosolic $\left[\mathrm{Ca}^{2+}\right]$ observed in cells exposed to FA could be explained by activation of calcium channels of plasma membrane. 

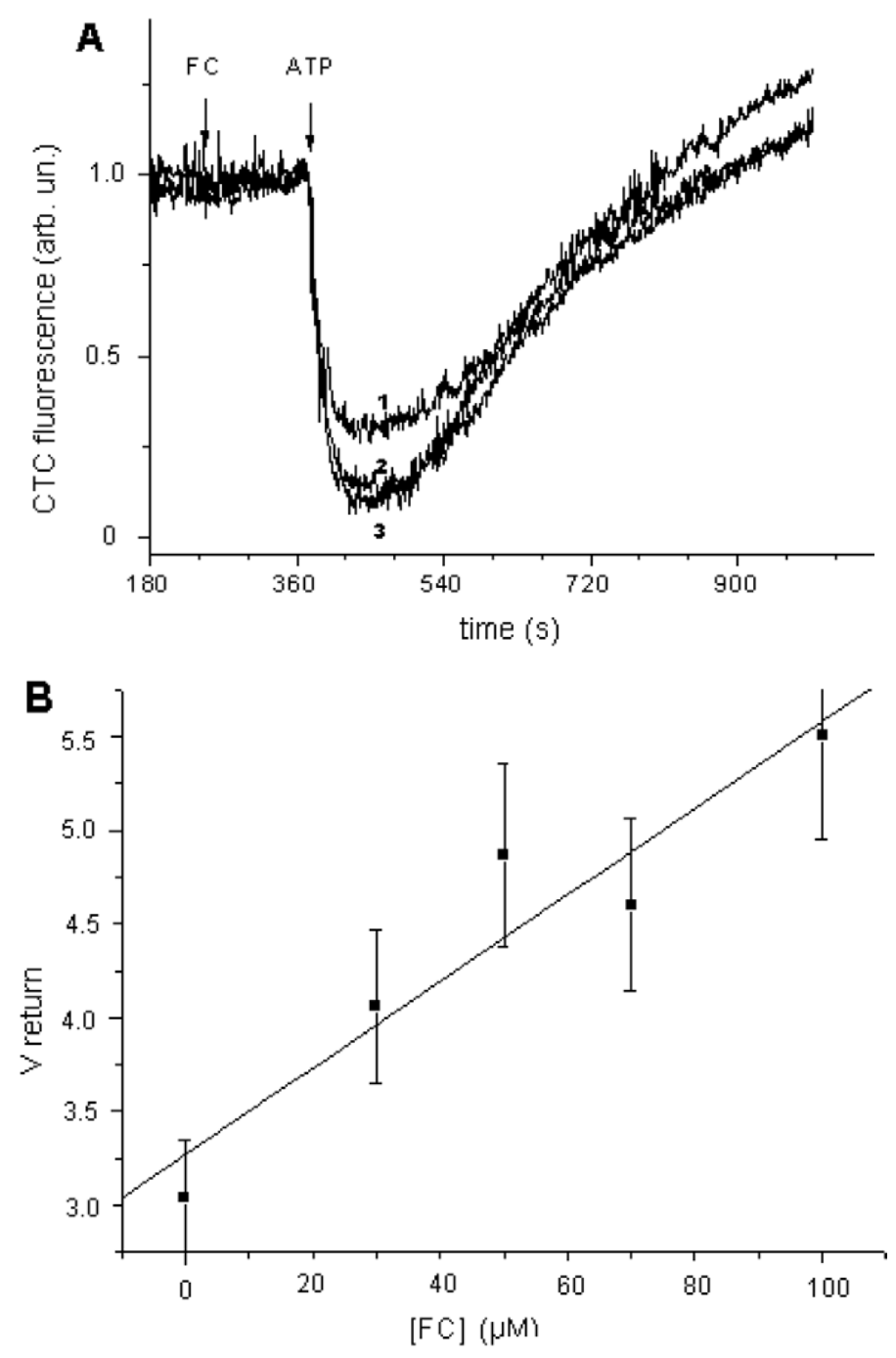

Fig. 6. Change of $\left[\mathrm{Ca}^{2+}\right]$ in ER of EATC in response to FC. (A) Influence of ATP, $4.5 \mu \mathrm{M}$; measured according to fluorescence of CTC. Traces: (1) control; (2) exposed to $10 \mu \mathrm{M}$ FC; (3) exposed to $20 \mu \mathrm{M}$ FC. (B) Dependence of the rate of $\mathrm{Ca}^{2+}$ return into ER on FC concentrations. FC increases the rate of $\mathrm{Ca}^{2+}$ return into ER in a dose-dependent manner. Summarized data: means $\pm 10 \%$ error $(n=3)$.

\subsection{Effects of FA on cardiomyocytes}

Figures 7A and 7B show changes in the cytosolic $\left[\mathrm{Ca}^{2+}\right]$ level and mitochondrial membrane potential, respectively, in four cardiomyocytes viewed using the confocal microscope. FA induced an increase in the basal level of cytosolic $\left[\mathrm{Ca}^{2+}\right]$ that either gave rise to calcium waves propagating over the surface of sarcoplasmic reticulum or markedly enhanced preexisting waves and their propagation rate. At the same time, there was a slow increase in mitochondrial membrane potential. Oligomycin and rotenone decreased TMRM fluorescence, indicating a decrease of mitochondrial membrane potential (Fig. 7B). Addition of oligomycin to control cells induced elevation of membrane potential, which was decreased by FCCP or rotenone (data not shown here). So it is possible that the increase of cytosolic $\left[\mathrm{Ca}^{2+}\right]$ 

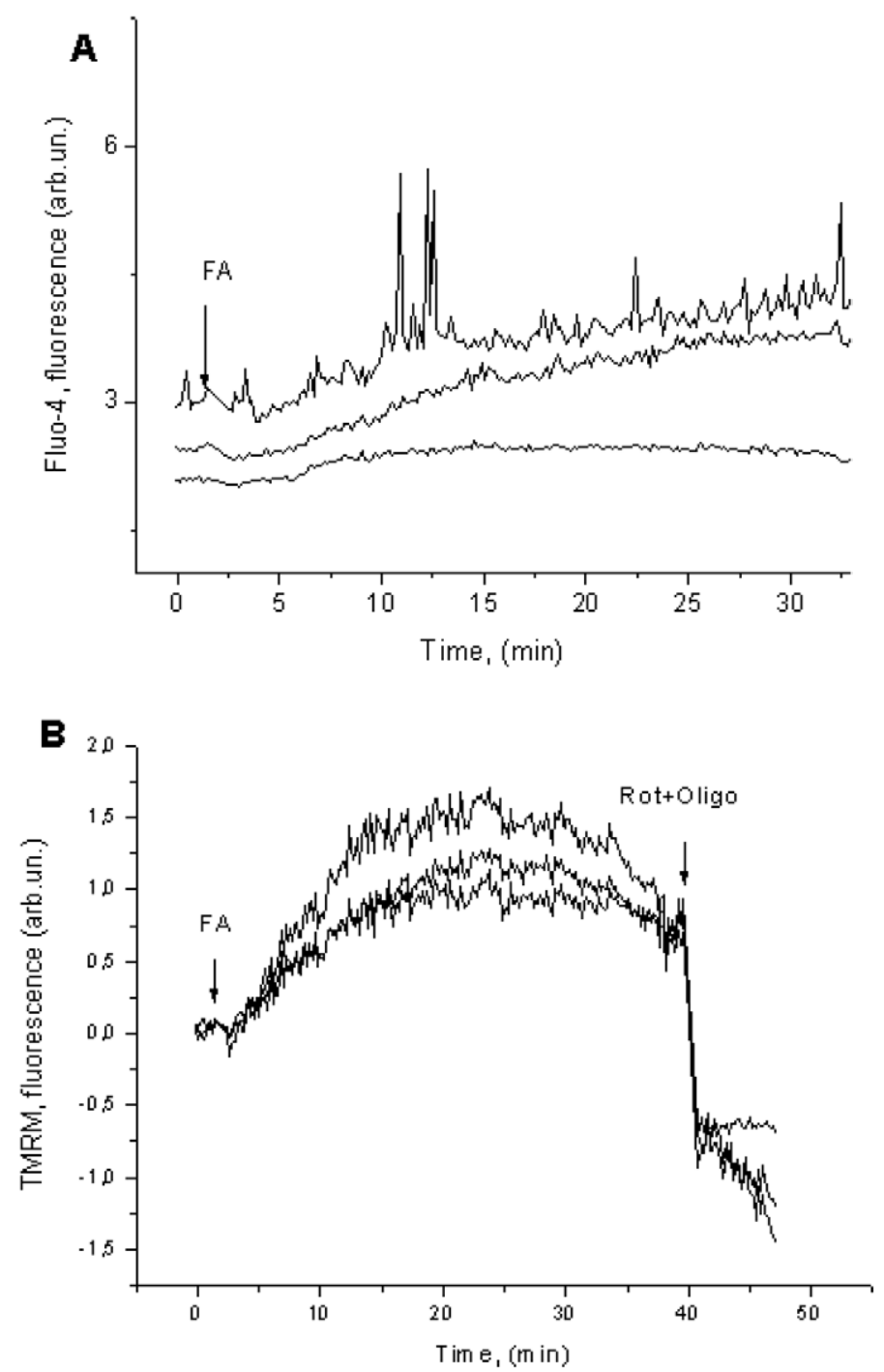

Fig. 7. Cardiomyocytes' response to FA, $10 \mathrm{mM}$. (A) Changes of cytosolic $\left[\mathrm{Ca}^{2+}\right]$ in three cardiomyocytes. (B) Changes of TMRM fluorescence in three cardiomyocytes in response to FA, $10 \mathrm{mM}$, and rotenone $(1 \mu \mathrm{g} / \mathrm{ml})$ with oligomycin $(1 \mu \mathrm{g} / \mathrm{ml})$ $(n=3)$.

is followed by its transport into mitochondria with subsequent inhibition of the proton ATPase and the resulting increase of membrane potential. Previously we demonstrated that isolated liver and heart mitochondria exhibited different responses upon accumulation of small amounts of calcium ions. The transport of $\mathrm{Ca}^{2+}$ enhanced the ADP-dependent oxidative phosphorylation in liver mitochondria but inhibited this response in heart mitochondria. These effects were accompanied by a slight decrease of liver mitochondrial membrane potential and increase in heart and brain mitochondrial membrane potential [23]. The mechanism of this phenomenon was subsequently shown to be associated with action of a $\mathrm{Ca}^{2+}$-dependent protein inhibiting $\mathrm{H}^{+}$-ATPase [24]. 
The increase of mitochondrial membrane potential under activation of $\mathrm{Ca}^{2+}$ entry into cytosol and/or under hypoxic conditions has also been demonstrated by other researchers, but only with cardiomyocytes and nervous cells of brain $[25,26]$.

\section{Discussion}

The data obtained in our experiments demonstrate that FA affects mitochondrial functions only in the presence of pyruvate as respiratory substrate. They also indicate that changes in the redox state of pyridine nucleotides and their leakage through the permeability transition pore could be critical events causing mitochondrial respiration disturbances and cell death. The pore opening is a reversible phenomenon, and prevention of oxidation and/or leakage of $\mathrm{NAD}(\mathrm{P}) \mathrm{H}$ from mitochondria could help to return them to the normal functional state. One of the natural ways to preventing oxidation of pyridine nucleotides could be utilization of alternative substrates and/or switching over to alternative metabolic pathways. For example, when succinate or glutamate were used as respiratory substrates, FA did not affect mitochondrial functions in vitro. This is consistent with our recently reported data on sodium glutamate as anaplerotic agent, which was capable to recover blood platelet functions affected by FA in vitro and in vivo [6]. Earlier it was hypothesized that under action of FA exogenic glutamate could be utilized via glutamate dehydrogenase or transamination pathways [27,28].

As for other alternative substrates, we suggest it could be the endogenous citrate that accumulates under intoxication with FA. It was demonstrated long ago that cytoplasmic aconitase was not inhibited by FC and accumulating citrate in liver cells can move from mitochondria to cytosol with its subsequent utilization by cytoplasmic aconitase and cytoplasmic NADP-dependent isocitrate dehydrogenase (cICDH) [29]. As for other tissues, in astroglial and microglial cultures the total ICDH activity was almost equally distributed between cytosolic and mitochondrial fractions, whereas in cultures of neurons and oligodendrocytes about $75 \%$ of total ICDH activity was present in the cytosolic fractions [30]. There is no corresponding data on the ratio of mitochondrial and cytoplasmic aconitase in the cells of the nervous system. We note however that in liver there is a similar ratio of isocitrate dehydrogenases [31] and that the activity of liver cytoplasmic aconitase is about $65 \%$ of the total aconitase activity in this organ [32]. The present findings may be explained by the existence of an effective metabolic pathway for utilization of citrate and synthesis of NADPH in nervous and other cells in the case of inhibition of mitochondrial aconitase. Such an alternative pathway would play an adaptive and positive role: oxygen consumption would be lowered because the resultant NADPH does not need to be oxidized in the respiratory chain and could be utilized for NADPH-dependent purposes, such as for anabolic reactions and heat generation, glutathion reduction and nitric oxide synthesis, vascular relaxation or constriction via reactive oxygen species generation [33-36]. It was noticed earlier that studies focusing on the pentosephosphate pathway as the sole source of NADPH may require re-evaluation depending on the overall metabolic capacity and substrate utilization of the particular tissue [33]. NADP ${ }^{+}$-dependent cICDH together with malic enzyme and nicotinamide nucleotide transhydrogenase contribute to the regeneration of NADPH required for the reduction of glutathione disulfide in brain mitochondria [37], but cICDH renders more than sevenfold generation of NADPH in comparison with malic enzyme in rat retina [33]. Direct evidence was obtained correlating the activities of $\mathrm{cICDH}$ and the maintenance of the cellular redox state, suggesting that cICDH plays an important role in cellular defence [38]. Moreover, the level of cytosolic NADPH can fundamentally affect potassium channels and intracellular calcium homeostasis [39]. We have demonstrated in in vitro studies that FA caused a slow increase of the cytosolic $\mathrm{Ca}^{2+}$ 
level in different cells. The observed primary increase of cytosolic $\left[\mathrm{Ca}^{2+}\right]$ in cells exposed to FA is indicative of activation of calcium channels of plasma membrane; the process is not affected by FA and therefore does not require ATP, at least in glial cells [40]. Assuming this event to be common for various cells, it could explain hypersensitivity of platelets to ADP under exposure to FA [6]. Elevation of cytosolic $\left[\mathrm{Ca}^{2+}\right]$ level in cardiomyocytes can trigger an enhancement of the functional activity of cardiomyocytes: the resulting increase of $\mathrm{Ca}^{2+}$ entry into endoplasmic reticulum could explain the data on enhanced amplitude and rate of $\mathrm{Ca}^{2+}$ waves in cardiomyocytes. Also, it is accompanied by elevation of their mitochondrial membrane potential, which is consistent with results of other researchers according to which the oxidative phosphorylation systems of heart and brain mitochondria had a special sensitivity to calcium ions. With regards to modulating effects of $\mathrm{Ca}^{2+}$ on mitochondrial bioenergetics, the "classical" activation by this ion of dehydrogenases of the TCA cycle could cause an increase of membrane potential and elevated production of NADH. These three dehydrogenases have been shown to be the targets for calcium ions: 2-oxoglutarate dehydrogenase and NAD-dependent isocitrate dehydrogenase are activated through an allosteric mechanism, while pyruvate dehydrogenase is activated through dephosphorylation by a $\mathrm{Ca}^{2+}$-dependent phosphatase [41]. The role for these dehydrogenases in the bioenergetic status of mitochondria under action of FA or FC remains to be elucidated, though in the case of mitochondrial aconitase blockade one can readily suppose 2-oxoglutarate dehydrogenase to be a critical point at which cells derive benefit from elevated $\left[\mathrm{Ca}^{2+}\right]$ upon addition of exogenous or by generation of endogenous sources of its substrate, 2-oxoglutarate. The question also remains as to the direction and extent the calcium balance in different cells is a point of modulation by various redox agents, which could reorient metabolic pathways by shifting NADH/NADPH ratio in mitochondria and cytosol. This is a subject of our current research.

\section{Acknowledgements}

This work was supported by the BioIndustry Initiative Program of the US Department of State, ISTC grant BII-2629.

\section{References}

[1] N.V. Goncharov, R.O. Jenkins and A.S. Radilov, Toxicology of fluoroacetate: a review, with possible directions for therapy research, J. Appl. Toxicol. 26 (2006), 148-161.

[2] P. Buffa, V. Guarriero-Bobyleva and R. Costa-Tiozzo, Metabolic effects of fluoroacetate poisoning in animals, Fluoride 6 (1973), 224-247.

[3] G.A. Perez and G. Frindt, The effect of fluorocitrate on urinary calcium and citrate excretion, Experientia 33 (1977), 741-742.

[4] A. Roy (Shapira), U. Taitelman and S. Bursztein, Evaluation of the role of ionized calcium in sodium fluoroacetate ('1080') poisoning, Toxicol. Appl. Pharmacol. 56 (1980), 216-220.

[5] I.M. Arena, Poisoning: Toxicology - Symptoms - Treatments, C.C. Thomas, Springfield, IL, 1970.

[6] I.V. Mindukshev, N.V. Goncharov, E.Yu. Shabanova, E.E. Ermolaeva, M.O. Mironova, A.S. Radilov, R.O. Jenkins and A.I. Krivchenko, A new method for studying platelets, based upon the low-angle light scattering technique. 3. Aggregation hypersensitivity of platelets (ADP agonist) and search for corrective agents, Spectroscopy 20 (2006), 57-66.

[7] R.H. Bowman, Inhibition of citrate metabolism by sodium fluoroacetate in the perfused rat heart and the effect on phosphofructokinase activity and glucose utilization, Biochem. J. 93 (1964), 13c-15c.

[8] T. Dohi and F. Murad, Effects of pyruvate and other metabolites on cyclic GMP levels in incubations of rat hepatocytes and kidney cortex, Biochim. Biophys. Acta 673 (1981), 14-25.

[9] W.M. Taylor, M. D'Costa, A. Angel and M.L. Halperin, Insulin-like effects of fluoroacetate on lipolysis and lipogenesis in adipose tissue, Can. J. Biochem. 55 (1977), 982-987. 
[10] A.J. Dickson and D.R. Langslow, Gluconeogenesis in isolated chicken hepatocytes, Biochem. Soc. Trans. 5 (1977), 983986.

[11] M.G. Cremer-Lacuara, J.L. Lacuara, M. Fiol de Cuneo and R.D. Ruiz, Substrate supply and function of isolated venous smooth muscle under anoxia and metabolic inhibition, Can. J. Physiol. Pharmacol. 58 (1980), 723-730.

[12] A.S. Fairhurst, R.E. Smith and B.M. Gal, The effects of fluorocompounds on oxidative phosphorylation, Biochem. Pharmacol. 1 (1958), 273-279.

[13] P. Buffa and I. Pasquali-Ronchetti, Biochemical lesions of respiratory enzymes and configurational changes of mitochondria in vivo. II. Early ultrastructural modifications correlated to the biochemical lesion induced by fluoroacetate, Cell Tissue Res. 183 (1977), 1-23.

[14] A. Corsi and A.L. Granata, Differential toxicity of fluoroacetate to heart, kidney and brain mitochondria of the living rat, Biochem. Pharmacol. 16 (1967), 1083-1089.

[15] V.V. Teplova, Yu.V. Evtodiyenko, E.I. Kholmukhamedov, N.G. Sergeyenko and N.V. Goncharov, [Effect of fluorocitrate on substrate oxidation and $\mathrm{Ca}^{2+}$ transporting systems of rat liver mitochondria], Tsitologia (Cytology, SPb) 34 (1992), 69-73. [Article in Russian.]

[16] Anon, Guide for the Care and Use of Laboratory Animals, National Academy Press, USA, 1996.

[17] W.C. Schneider, Intracellular distribution of enzymes, J. Biol. Chem. 176 (1948), 259-266.

[18] G.M. Frank, M.N. Kondrashova, E.N. Mokhova and Yu.S. Rotenberg, eds, [Guide to study biological oxidation with polarography] Moscow, Nauka, 1973 [in Russian].

[19] T. Powell, D.A. Terrar and V.W. Twist, Electrical properties of individual cells isolated from adult rat ventricular myocardium, J. Physiol. 302 (1980), 131-153.

[20] P. Arslan, F. Divirgilio, M. Betzame, R. Tsien and T. Pozzan, Cytosolic $\mathrm{Ca}^{2+}$ homeostasis in Ehrlich and Yoshida carcinomas, J. Biol. Chem. 260 (1985), 2719-2727.

[21] V.P. Zinchenko, V.V. Teplova and Iu.V. Evtodienko, [Kinetics of oxygen consumption, luminescence of pyridine nucleotides and the cyanine dye $3^{\prime}, 3^{\prime}$-diethylthiodicarbocyanine iodide after energizing Ehrlich ascites carcinoma cells with glucose], Biull. Eksp. Biol. Med. 94 (1982), 69-72. [Article in Russian.]

[22] V.P. Zinchenko, L.P. Dolgacheva, E.L. Nikiforov and Yu.V. Kim, Regulation of purinoceptor-induced $\mathrm{Ca}^{2+}$ changes in mitochondria of Ehrlich ascites tumor cells, in: Calcium Signaling, M. Morad and P. Kostyuk, eds, IOS Press, NATO Science Series, Series 1: Life and Behavioral Sciences, Vol. 331, 2001, pp. 217-225.

[23] Iu.S. Karadzhov, L.Iu. Kudzina and V.P. Zinchenko, [Effect of Ca ions on the transmembrane electric potential, synthesis and hydrolysis of ATP in brain mitochondria], Biofizika 33 (1988), 77-82. [Article in Russian.]

[24] M.J. Hubbard and N.J. McHugh, Mitochondrial ATP synthase F-1-beta-subunit is a calcium-binding protein, FEBS Lett. 391 (1996), 323-329.

[25] D.B. Zorov, C.R. Filburn, L.O. Klotz, J.L. Zweier and S.J. Sollott, Reactive oxygen species (ROS)-induced ROS release: a new phenomenon accompanying induction of the mitochondrial permeability transition in cardiac myocytes, J. Exp. Med. 192 (2000), 1001-1014.

[26] B. Khodorov, Glutamate-induced deregulation of calcium homeostasis and mitochondrial dysfunction in mammalian central neurons, Prog. Biophys. Mol. Biol. 86 (2004), 279-351.

[27] H.L. Yu, R. Giammarco, M.B. Goldstein, D.J. Stinebaugh and M.L. Halperin, Stimulation of ammonia production and excretion in the rabbit by inorganic phosphate: study of control mechanisms, J. Clin. Invest. 58 (1976), 557-564.

[28] C. Liang, Metabolic control of circulation: effects of iodoacetate and fluoroacetate, J. Clin. Invest. 60 (1977), 61-69.

[29] S.R. Max and J.L. Purvis, Energy-linked incorporation of citrate into rat liver mitochondria, Biochem. Biophys. Res. Commun. 21 (1965), 587-594.

[30] T. Minich, S. Yokota and R. Dringen, Cytosolic and mitochondrial isoforms of NADP ${ }^{+}$-dependent isocitrate dehydrogenases are expressed in cultured rat neurons, astrocytes, oligodendrocytes and microglial cells, J. Neurochem. 86 (2003), 605-614.

[31] T.I. Rakhmanova and T.N. Popova, Regulation of 2-oxoglutarate metabolism in rat liver by NADP-isocitrate dehydrogenase and aspartate aminotransferase, Biochemistry (Moscow) 71 (2006), 211-217.

[32] S.G. Konstantinova and E.M. Russanov, Aconitase activity in rat liver, Comp. Biochem. Physiol. B: Biochem. Mol. Biol. 113 (1996), 125-130.

[33] B.S. Winkler, N. De Santis and F. Solomon, Multiple NADPH-producing pathways control glutathione (GSH) content in retina, Exp. Eye Res. 43 (1986), 829-847.

[34] V. Bobyleva, N. Kneer, M. Bellei, D. Battelli and H.A. Lardy, Concerning the mechanism of increased thermogenesis in rats treated with dehydroepiandrosterone, J. Bioenerg. Biomembr. 25 (1993), 313-321.

[35] T.J. Lee and J.G. Yu, L-Citrulline recycle for synthesis of NO in cerebral perivascular nerves and endothelial cells, Ann. N.Y. Acad. Sci. 962 (2002), 73-80.

[36] S.A. Gupte and M.S. Wolin, Hypoxia promotes relaxation of bovine coronary arteries through lowering cytosolic NADPH, Am. J. Physiol. Heart Circ. Physiol. 290 (2006), H2228-2238. 
[37] R. Vogel, H. Wiesinger, B. Hamprecht and R. Dringen, The regeneration of reduced glutathione in rat forebrain mitochondria identifies metabolic pathways providing the NADPH required, Neurosci. Lett. 275 (1999), 97-100.

[38] S.H. Lee, S.H. Jo, S.M. Lee, H.J. Koh, H. Song, J.W. Park, W.H. Lee and T.L. Huh, Role of NADP ${ }^{+}$-dependent isocitrate dehydrogenase $\left(\mathrm{NADP}^{+}-\mathrm{ICDH}\right)$ on cellular defense against oxidative injury by gamma-rays, Int. J. Radiat. Biol. 80 (2004), 635-642.

[39] M.S. Wolin, M. Ahmad and S.A. Gupte, Oxidant and redox signaling in vascular oxygen sensing mechanisms: basic concepts, current controversies, and potential importance of cytosolic NADPH, Am. J. Physiol. Lung Cell Mol. Physiol. 289 (2005), L159-173.

[40] X.Y. Lian and J.L. Stringer, Astrocytes contribute to regulation of extracellular calcium and potassium in the rat cerebral cortex during spreading depression, Brain Res. 1012 (2004), 177-184.

[41] J. McCormack, A. Halestrap and R. Denton, Role of calcium ions in regulation of mammalian intramitochondrial metabolism, Physiol. Rev. 70 (1990), 391-425. 


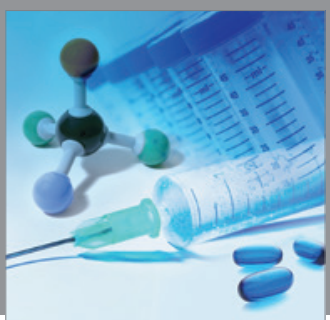

International Journal of

Medicinal Chemistry

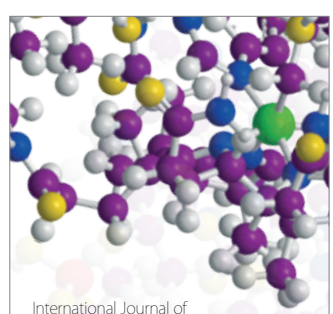

Carbohydrate Chemistry

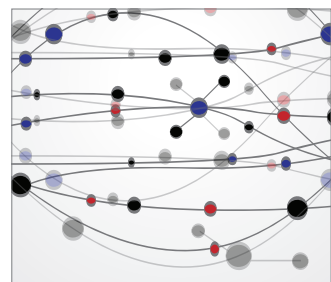

The Scientific World Journal
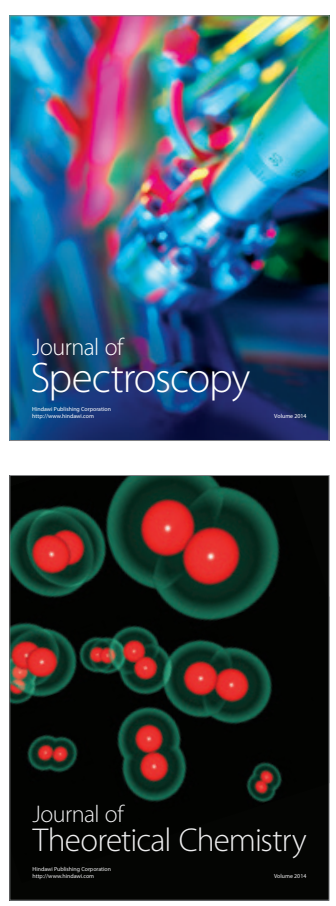
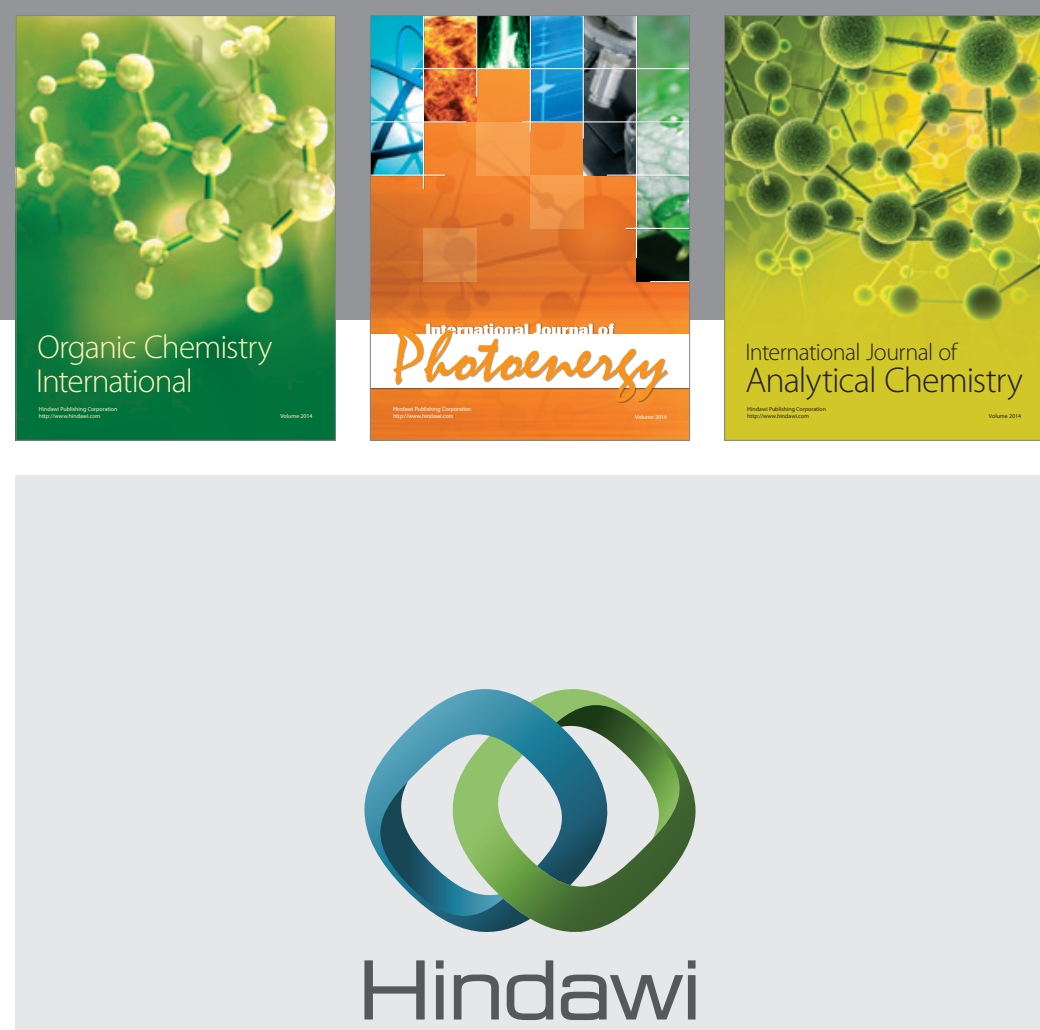

Submit your manuscripts at

http://www.hindawi.com
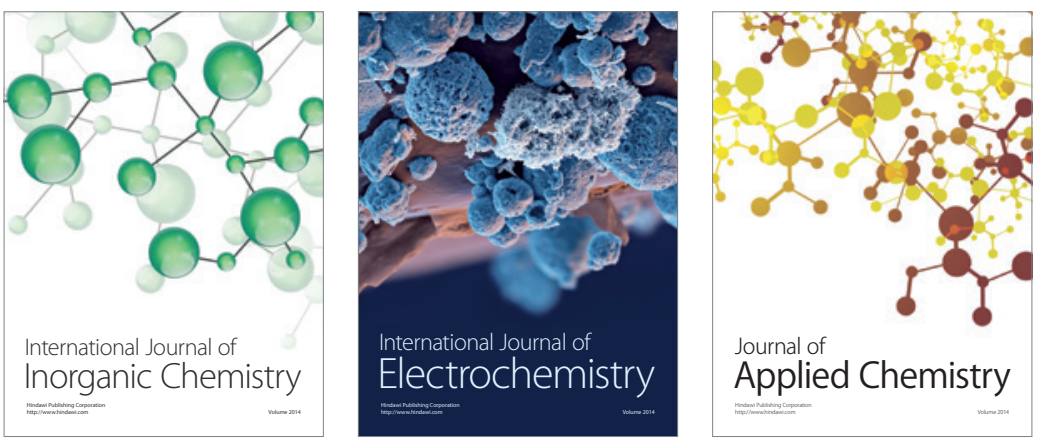

Journal of

Applied Chemistry
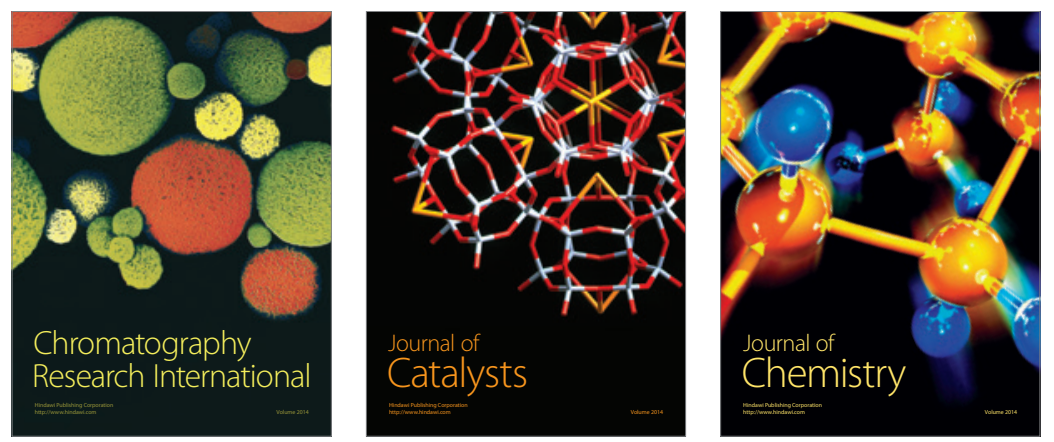
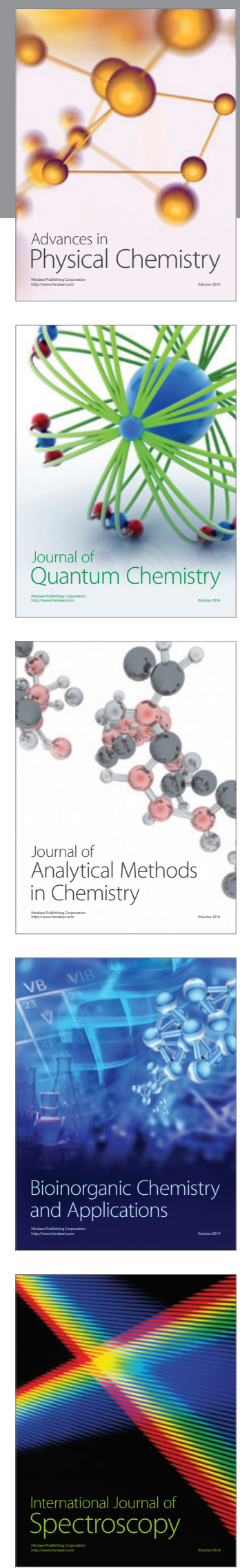